\title{
An Analysis of Membrane Mirrors For Use in Large Aperture Imaging Systems
}

\author{
Kevin W. Peters, Jonathan A. Bishop, and James M. Wilkes \\ Air Force Research Laboratory \\ AFRL/VSSS \\ 3550 Aberdeen Ave. SE \\ Kirtland AFB, NM 87117 \\ (505) 853-3524 \\ Email: peterskw@plk.af.mil
}

\begin{abstract}
The finite element model was used to determine the surface figure of membrane mirrors. The Poisson's ratio of the membrane was varied to determine the most desirable materials. It was found that materials with a Poisson's ratio of 0.45 or greater are desirable. Membranes mirrors alone are poor imaging surfaces, and the effects of radial boundary displacement are investigated. It was found that radial boundary displacement can reduce the rms wavefront error of a 42 inch diameter $f / 1.9$ mirror by $78.7 \%$. A 42 inch diameter $\mathbf{f} / \mathbf{1 . 0 6}$ membrane mirror would have the rms wavefront error reduced by $65.6 \%$. The primary on-axis and off-axis aberration was found to be $3^{\text {rd }}$ order spherical aberration for all f-numbers and at all radial displacements.
\end{abstract}

\section{TABLE OF CONTENTS}

1. INTRODUCTION

2. MATERIAL PROPER TIES

3. RADIAL BOUNDARY DISPLACEMENT

4. DISCUSSION

5. CONCLUSIONS

\section{INTRODUCTION}

For space based optical imaging systems, large aperture primary mirrors are desirable due to their capability to provide high resolution imaging. To achieve the required optical quality, traditional mirrors must be extremely heavy and very large which causes these mirrors to be very expensive or impossible to launch into space. Segmenting the primary mirror, using a sparse array, and inflating a membrane mirror are three possible alternatives to conventional mirrors[1]. All of these could achieve the high resolutions desired yet be more feasible for space usage. The advantages of a membrane mirror are its low weight and the potential to achieve extremely large diameters of 20 meters or larger. However, the optical performance of these membranes is largely unknown. In this work, the finite element model was utilized to determine the shape of a membrane surface under uniform pressure. This shape was then used to analyze the optical performance of a membrane mirror to determine its feasibility as an imaging surface. This paper will explore how the material properties of the membrane affect performance, and investigate the effects of radial boundary displacement. This investigation will include an analysis of the different aberrations present for an infinite conjugate as well as examine the off-axis performance of these mirrors.

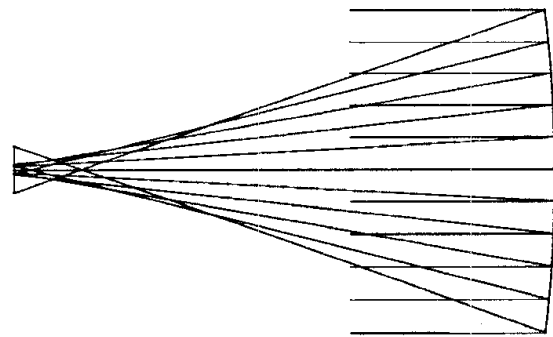

(a)

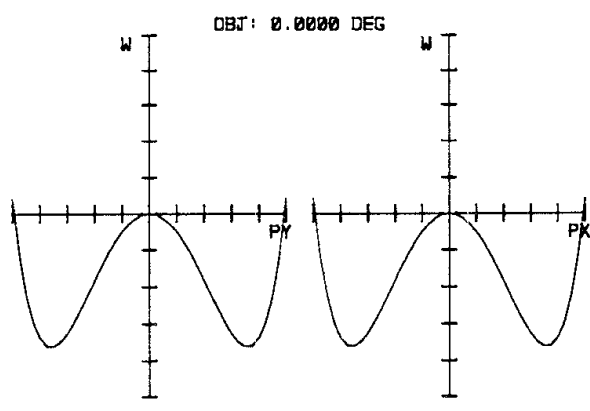

(b)

Figure 1 (a) Ray trace of typical membrane mirror and (b) OPD plot of $\mathrm{f} / 1.9$ membrane (Scale is $+/-5000$ waves).

The behavior of membranes subjected to a uniform pressure has been studied for many years[2-4]. In one such study, Stevens [4] gives the following formula for the displacement of a membrane:

$$
w(r)=b\left(1-c_{1} \frac{r^{2}}{a^{2}}-c_{2} \frac{r^{5}}{a^{5}}\right)
$$

where $b$ is the maximum displacement, $c_{1}$ is $0.9, c_{2}$ is 0.1 , and $a$ is the aperture radius. The maximun displacement, $b$, is a function of the $f$-number and the aperture radius. Figure 
1 is a ray trace of typical membrane mirror with its corresponding optical path difference (OPD) plot. Stevens' formula suggests that membranes under uniform pressure will have an aspheric shape which departs from an ideal parabola. Due to this aspheric shape, membrane mirrors will be extremely poor imaging surfaces especially at small f-numbers and large diameters. These surfaces would be excluded from use in imaging systems, even those systems which include adaptive optics. Nevertheless, membrane mirrors could be useful if the surface could be significantly improved. One technique to improve the surface figure is to apply a uniform radial boundary displacement to the membrane mirror. The finite element model allows for the study of a membrane under these conditions.

\section{MATERIAL PROPERTIES}

Materials used as membrane mirrors must meet certain requirements. First, the materials must have an extremely small thickness on the order of several hundred microns. This allows the membrane to be deformed without requiring unreasonable pressures to be exerted. Second, the material must be highly reflective or be capable of maintaining a highly reflective coating of a material such as aluminum or silver. Third, the surface smoothness must meet optical requirements. Finally, since membranes will require radial boundary displacements, the materials must be capable of withstanding the forces applied.

The effect of the Poisson's ratio ( $v$ ) on the membrane mirror was investigated. Poisson's ratio for a material is the ratio of its transverse contraction to its elongation when subjected to a tensile stress. Variations in Young's Modulus were not studied since basic theory and nonlinear finite element analysis both show that a change in this parameter has the effect of only changing the pressures required and does not change the shape of the imaging surface. Also, because the membrane is axisymmetric, the shear modulus is irrelevant since there is no shear strain. However, a change in $v$ does have an effect on the surface structure.

Using the finite element model to determine the theoretical surface structure, it was found that as $v$ increases, the optical performance of the membrane improves. Poisson's ratio was varied from 0.25 to 0.49 . Since the membrane is a continuum, $v$ cannot exceed 0.5 . The calculated rms (to mean) wavefront error of a 42 inch diameter f/1.11 mirror decreases from 2740 waves at $0.55 \mu$ for $v=0.25$ to 1814 waves for $v=0.49$. For the remainder of this paper, the rms wavefront error will be referenced to the mean and will be in waves at $0.55 \mu$ unless otherwise noted. Figure 2 plots the rms wavefront error at different Poisson's ratios. From this analysis it was determined that the ideal material used for the membrane mirror should have a Poisson's ratio of 0.45 or greater.

At $v=0.45$, the rms wavefront error for the 42 inch diameter $\mathrm{f} / 1.11$ mirror is 1814 waves at $0.55 \mu$ which is in good agreement with Stevens' formula that predicts an rms wavefront error of 1850 waves, a $2 \%$ difference. The primary on-axis aberration is $3^{\text {rd }}$ order spherical aberration, and this aberration dominates over primary coma and astigmatism even at a field of view of four degrees. It should be noted that the on-axis performance is also affected by higher order spherical aberration as well. 1814 waves is an unacceptable amount of aberration for an imaging surface since even a few waves of aberration causes a significant degradation of the image. One possible method for reducing these aberrations is to apply a uniform radial boundary displacement to the membrane which is the subject of the next section.

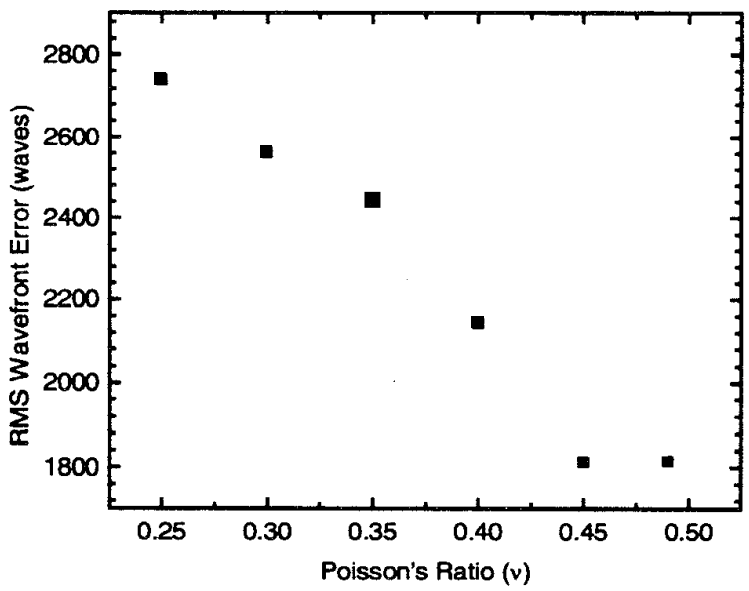

Figure 2 RMS Wavefront Error at $0.55 \mu$ as a function of Poisson's Ratio.

\section{RADIAL BOUNDARY DISPLACEMENT}

Stevens' formula does not predict the behavior of membrane when the radial boundary is displaced. Therefore, the finite element model was used to predict the surface shape when the membrane is subjected to a uniform radial boundary displacement. The finite element model is a quarter-disk consisting of 304 eight-noded quadrilateral membrane elements and 16 six-noded triangular membrane elements. In all cases, the model was initiated from planar configuration. In order to give a well-conditioned stiffness matrix for small deflections, transverse springs were attached to each node. The model was inflated to a small pressure (5\%-10\% of final pressure), and the boundary was displaced as called for by each case. The springs were then removed, and the pressure was increased as necessary to obtain the desired center deflection.

All the mirrors in this study had a 42 inch diameter and Poisson's ratio was assumed to be 0.4 . The wavelength used for all calculations is $0.55 \mu$. We studied the effects of a radial boundary displacement for mirrors that had an fnumber of $f / 1.9, f / 1.44$, and $f / 1.06$. To maintain a constant f-number for comparison, the maximum deflection along the $z$ axis was kept constant as the radial boundary was displaced by increasing the pressure on the membrane as is seen in figure 3 .

Initially, the case where the radial displacement was applied before the membrane inflation and the case where the membrane was first inflated then the radial boundary was 
displaced were both studied. However, it was discovered that both cases give the same result for the surface figure. Therefore, just the former case is presented here.

To analyze the separate aberrations present, the zernike polynomials are used to describe the wavefront. A discussion of these polynomials can be found in any lens design text[5], and a brief description is given here merely for convenience. The zernike polynomials are an orthogonal set of polynomials where each polynomial represents a different aberration. The wavefront variance is defined as [5]

$$
\sigma_{w}^{2}=\frac{1}{\pi} \int_{0}^{1} \int_{0}^{2 \pi}\left(W(\rho, \theta)-W_{\text {ovg }}\right)^{2} \rho d \rho d \theta
$$

where $\rho, \theta$ are polar coordinates in the entrance pupil, $\rho$ is normalized, and $W(\rho, \theta)=z_{m n} U_{m n}(\rho, \theta)$ where $U_{m n}(\rho, \theta)$ is the $\mathrm{mn}$ zernike polynomial and $\mathrm{z}_{\mathrm{mn}}$ is the $\mathrm{mn}$ zernike coefficient. The zernike polynomials which describe $3^{\text {rd }}$ and $5^{\text {th }}$ spherical aberration are given by, respectively[5]

$$
\begin{gathered}
U_{42}(\rho, \theta)=6 \rho^{4}-6 \rho^{2}+1 \\
U_{63}=20 \rho^{6}-30 \rho^{4}+12 \rho^{2}-1
\end{gathered}
$$

Since the zernike polynomials are orthogonal, the wavefront variance can be written as

$$
\sigma_{w}^{2}=\sum_{m} \sum_{n} \sigma_{m n}^{2}
$$

where $\sigma_{\mathrm{nm}}{ }^{2}$ is the contribution from each aberration. The contribution for $3^{\text {rd }}$ and $5^{\text {th }}$ order spherical aberration is $\sigma_{42}^{2}=z_{42}^{2} / 5$ and $\sigma_{63}^{2}=z_{63}^{2} / 7$, respectively. The remainder of the aberrations are left to the reader.

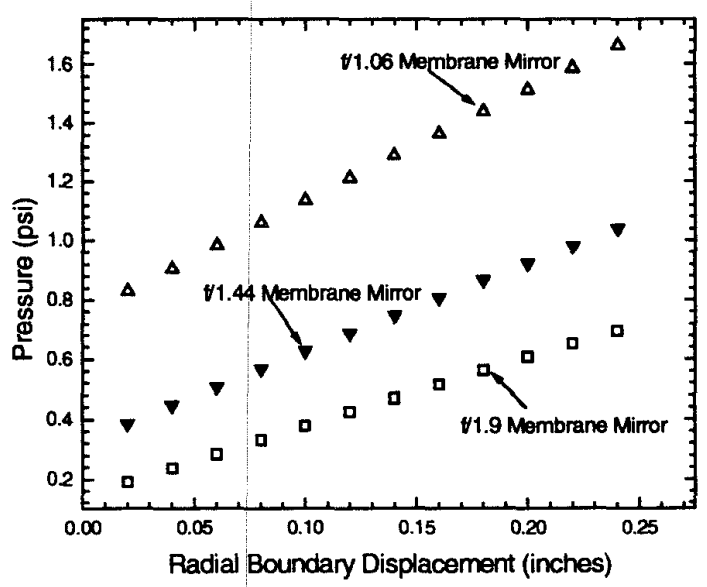

Figure 3 A plot of the pressure required to maintain a constant $f$-number as a function of radial boundary displacement.

\section{F/l.9 Membrane Mirror}

For the $\mathrm{f} / 1.9$ membrane mirror, a small radial displacement of 0.02 inches reduces the rms wavefront error by $22.7 \%$, from 1135 waves to 877 waves. The primary on-axis aberration is $3^{\text {rd }}$ order spherical aberration, and the calculated $\mathrm{z}_{42}$ zernike coefficient reduces from 2450.5 to 1965.3 , a $22.6 \%$ reduction. The $5^{\text {th }}$ order spherical aberration is also reduced by the radial boundary displacement. The $z_{63}$ zernike coefficient is reduced by $46.1 \%$ for a radial displacement of 0.02 inches and by $63 \%$ for a radial displacement of 0.04 inches while the rms wavefront error is only reduced by $37.6 \%$. This result initially caused us to believe that the higher order aberrations could be eliminated and the primary aberrations could be reduced to an acceptable level by increasing the radial displacement. However, this prediction turned out to be false as the primary and higher order aberrations reached a limit of correction. In other words, the point was reached were an additional radial boundary displacement did not provide any additional optical improvement of the membrane mirror.

Nevertheless, this should not be thought of as a negative result since the aberrations of this surface are significantly reduced. For a uniform radial boundary displacement of 0.24 inches, the rms wavefront error is reduced by $78.1 \%$ to 249 waves while $z_{42}$ is reduced by $78.05 \%$ and $z_{63}$ is reduced by $90.6 \%$. Figure 4 shows the improvement of the aberrations as the radial boundary displacement is increased. As a function of the radial displacement, the reduction in the rms wavefront error for the $\mathrm{f} / 1.9$ mirror is given by

$$
w=242.15+876.33 e^{-14.86 r}
$$

where $w$ is the rms wavefront error in waves and $r$ is the radial boundary displacement in inches. The $3^{\text {rd }}$ and $5^{\text {th }}$ order spherical aberration zernike coefficients follow a similar exponential decay given by

$$
\begin{aligned}
& z_{42}=542.0+1961.6 e^{-14.83 r} \\
& z_{63}=14.25+167.25 e^{-29.2 r}
\end{aligned}
$$

where $z_{42}$ is the zernike coefficient for $3^{\text {rd }}$ order spherical aberration and $z_{63}$ is the zernike coefficient for $5^{\text {th }}$ order spherical aberration. Equations (6), (7), (8) are best fit exponential decay curves to the data points calculated by the finite element model, see figure 4.

From these relationships, it becomes obvious that for small $r$, a slight increase in $r$ gives rise to a significant decrease in the aberrations. As $r$ increases, a slight change in $r$ results in a less significant improvement in performance. These relationships also show that a limit is reached where the aberrations no longer decrease. The minimum possible rms wavefront error predicted by (6) is 242.15 waves. By comparison, a f/1.9 sphere would have an $\mathrm{rms}$ wavefront error of 41 waves. The exponential decay constant in (8) is larger than in (6) and (7) and accounts for the more rapid decrease of the $5^{\text {th }}$ order spherical aberration. The $3^{\text {rd }}$ order 
spherical aberration is decreases at a similar rate as the rms wavefront error. This is to be expected since $3^{\text {rd }}$ order spherical aberration is the primary aberration.

Off-axis, the primary aberration is still $3^{\text {rd }}$ order spherical aberration even at a $4^{\circ}$ field of view (FOV) yet tilt, astigmatism, and primary coma are all present. The off-axis aberrations are also reduced, but not as significantly as the on-axis aberrations. The $z_{10}$ zernike coefficient corresponding to tilt reduces by a mere $1.04 \%$ to 384.5 . The $\mathrm{z}_{22}$ coefficient corresponding to astigmatism reduces a scant $2.2 \%$ while $z_{31}$, corresponding to primary coma, reduces $1.5 \%$. Therefore, radial boundary displacement does not have a profound effect on off-axis performance.

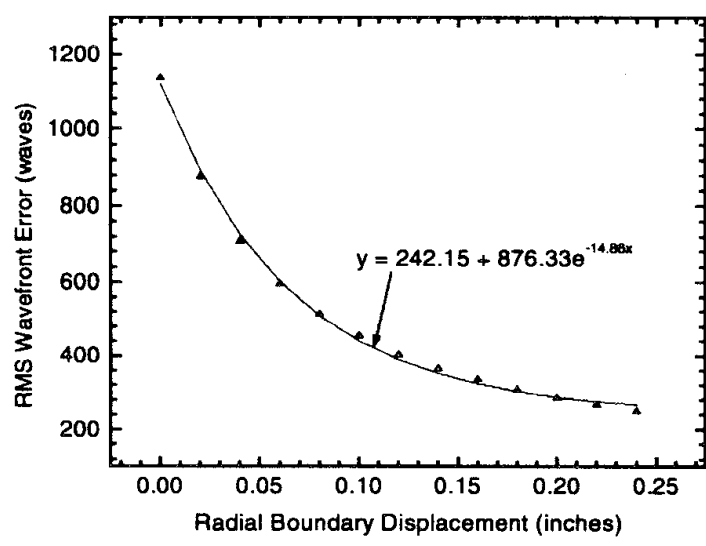

(a)

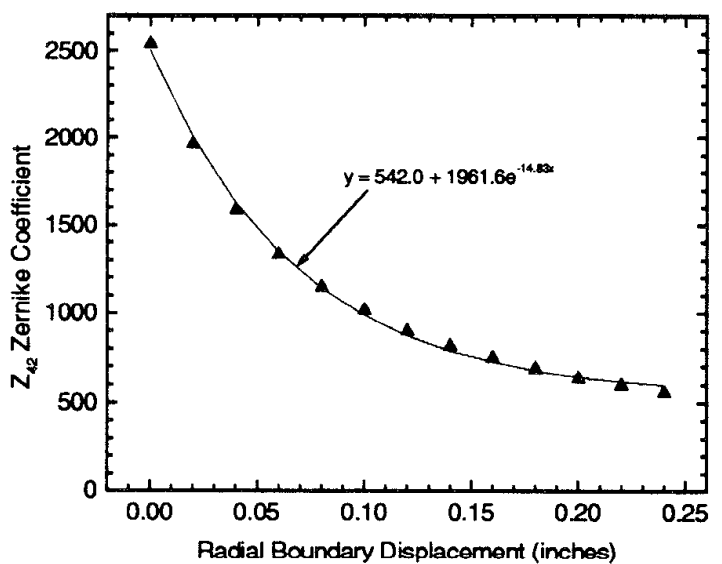

(b)

Figure 4 A plot of (a) RMS wavefront error and (b) $Z_{42}$ zernike coefficient versus radial boundary displacement for an $\mathrm{f} / 1.9$ membrane mirror.

\section{F/1.44 Membrane Mirror}

The exponential decay of the rms wavefront error seen in the case of the $f / 1.9$ mirror also occurs for the $f / 1.44$ membrane mirror. However, some important differences do take place. As in the previous case, a small radial displacement of 0.02 inches on the $\mathrm{f} / 1.44$ mirror reduces the rms wavefront error by $15.0 \%$ from 1554 waves to 1320 waves. The $\mathrm{z}_{42}$ coefficient is also reduced by $15.0 \%$. The $\mathrm{z}_{63}$ zernike coefficient is reduced by $22.8 \%$ while a slightly larger radial displacement of 0.04 inch reduces $z_{63}$ by $50.7 \%$. A larger radial displacement of 0.24 inches decreases the rms wavefront error by $67.0 \%, \mathrm{z}_{42}$ by $66.9 \%$, and $\mathrm{z}_{63}$ by $88.0 \%$. Figure 5 shows the decrease in aberrations as a function of the radial boundary displacement. The exponential decay for the rms wavefront error, $\mathrm{z}_{42}$, and $\mathrm{z}_{63}$ are given by

$$
\begin{gathered}
w_{f / 1.44}=428.6+1110.2 e^{-10.23 r} \\
z_{42(f / 1.44)}=959.0+2484.9 e^{-10.2 r} \\
z_{63(f / 1.44)}=31.12+226.9 e^{-i 9.6 r}
\end{gathered}
$$

where $r$ is the radial boundary displacement in inches. As before, (9), (10), and (11) are best fit exponential decay curves. From (9), the minimum rms wavefront error for an f/1.44 membrane mirror is 428.6 waves, a $72.4 \%$ decrease. For the f/1.9 mirror, the rms wavefront error could be reduced by $78.7 \%$. For the $\mathrm{f} / 1.44$ mirror, the maximum reduction of $z_{42}$ is $72.4 \%$ while $z_{63}$ can be reduced by $88.7 \%$. The exponential decay constant in (9), (10), and (11) is smaller than in (6), (7), and (8), respectively. This suggests that a larger radial displacement is needed for an $\mathrm{f} / 1.44$ mirror to achieve the desired improvement in performance.

\section{F/1.06 Membrane Mirror}

Faster mirrors are desirable for large aperture imaging systems since these mirrors reduce the total optical track. Therefore, the finite element model was used to investigate the performance of an $\mathrm{f} / 1.06$ mirror. For this mirror with no radial tension, the rms wavefront error was found to be 2154 waves. However, with a radial boundary displacement of 0.24 inches, the rms wavefront error could be reduced to 1015 waves, a significant improvement. The rms wavefront error exhibits an exponential decay for the $f / 1.06$ membrane mirror similar to the previous two cases. The rms wavefront error as a function of radial boundary displacement is described by

$$
w_{f / 1.06}=741.4+1407.4 e^{-6.74 r}
$$

Equation (12) suggests that the maximum decrease in the rms wavefront error is $65.6 \%$ to 741.4 waves. This result suggests that as the focal length of the membrane mirror increases, radial boundary displacement is capable of higher correction to the rms wavefront error. Therefore, the $f / 1.9$ mirror can achieve a $78.7 \%$ reduction in $\mathrm{mms}$ wavefront error while the faster mirrors cannot achieve this large of a reduction. In addition to this, the faster mirror requires a larger radial boundary displacement to achieve the desired improvement in optical performance since the exponential decay constant in (12) is smaller than (6) and (9). Figure 6 summarizes the results for an $\mathrm{f} / 1.06$ mirror. 


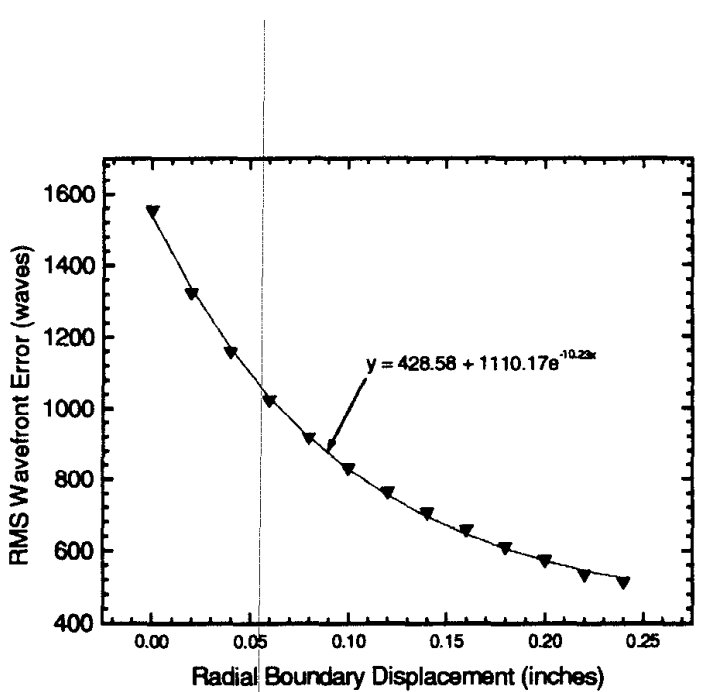

(a)

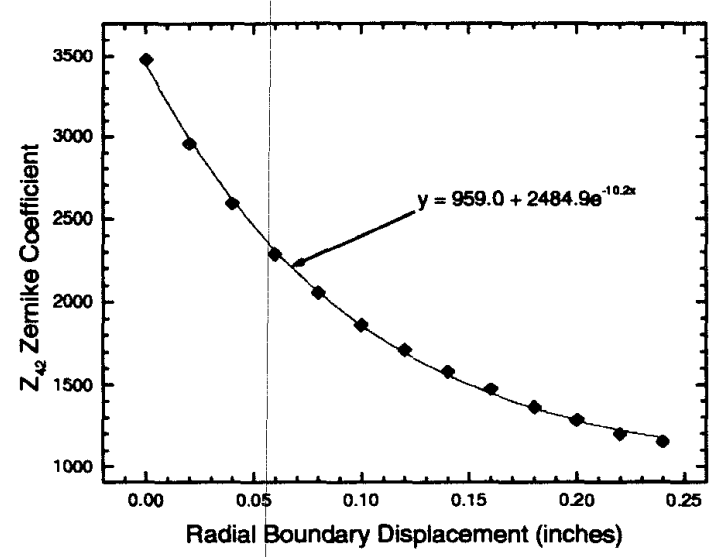

(b)

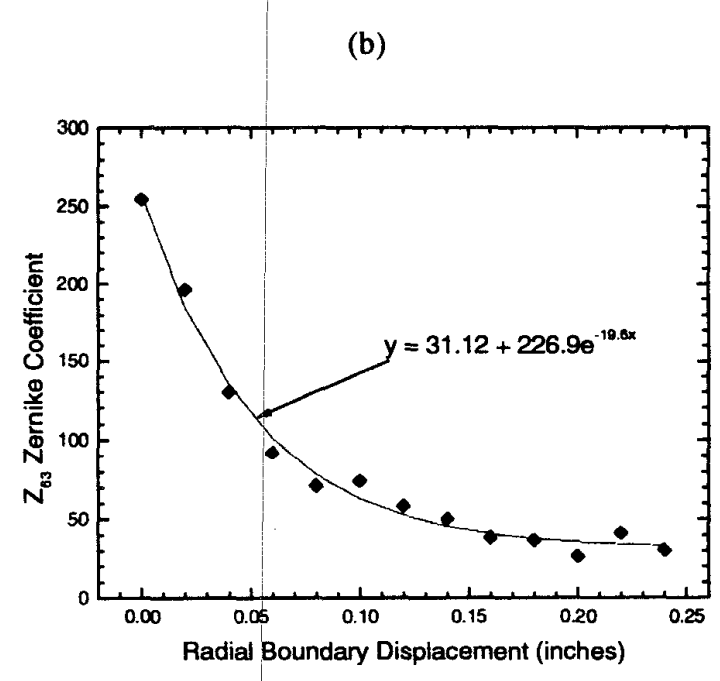

(c)

Figure 5 (a) RMS wavefront error, (b) $Z_{42}$ coefficient, and

(c) $Z_{63}$ coefficient as a function of radial boundary displacement for an $\mathrm{f} / 1.44$ membrane mirror.

\section{DISCUSSION}

The results presented here show the capability of radial boundary displacement to improve the optical performance of a 42 inch membrane mirror. However, one is interested in the performance of membrane mirrors over a broader range of aperture diameters. Since the finite element model has arbitrary units, equations (6), (9), and (12) can be used to generalize the rms wavefront error for the case of no radial displacement and maximum correction. For any aperture diameter, the predicted rms wavefront error for an f/1.9 membrane mirror with no radial tension (i.e. $r=0$ ) is given by

$$
\begin{aligned}
w_{f / 1.9} & =1118.5 \cdot \frac{D}{1066.8} \\
& =1.0485 \cdot D
\end{aligned}
$$

where $D$ is the aperture diameter in millimeters and $w_{f / 1.9}$ is the rms wavefront error in waves at $0.55 \mu$. The minimum rms wavefront error possible for an $\mathrm{f} / 1.9$ membrane mirror with radial boundary displacement is

$$
\begin{aligned}
w_{\min f / 1.9} & =242.15 \cdot \frac{D}{1066.8} \\
& =0.227 \cdot D
\end{aligned}
$$

where $\mathrm{D}$ is in millimeters. These relationships are given below for the $f / 1.44$ and $f / 1.06$ membrane mirrors, respectively.

$$
\begin{gathered}
w_{f / 1.44}=1.4424 \cdot D \\
w_{\min f / 1.44}=0.4018 \cdot D \\
w_{f / 1.06}=2.014 \cdot D \\
w_{\min f / 1.06}=0.695 \cdot D
\end{gathered}
$$

where, as before, $\mathrm{D}$ is the aperture diameter in millimeters. Therefore, a 42 meter diameter $\mathrm{f} / 1.9$ membrane would have an rms wavefront error of over 44,000 waves without radial tension, and achieve a minimum rms wavefront error of 9534 waves with radial boundary displacement. Furthermore, diffraction limited performance can not be achieved with radial boundary displacement even at small diameters of $4 \mathrm{~cm}$ where an $\mathrm{f} / 1.9$ membrane would have a minimum rms wavefront error of 9.08 waves. This could cause one to immediately conclude that membrane mirrors are not useful. However, this would be a premature conclusion for several reasons which will be discussed in the next paragraph.

First, membranes mirrors could be useful in the microwave and radar regimes where the radial boundary displacement reduction of the rms wavefront error by $66 \%$ or greater would improve performance to acceptable levels. For example, a 42 meter diameter f/1.06 membrane would have a minimum rms wavefront error of 0.16 waves at $\lambda=10 \mathrm{~cm}$. 


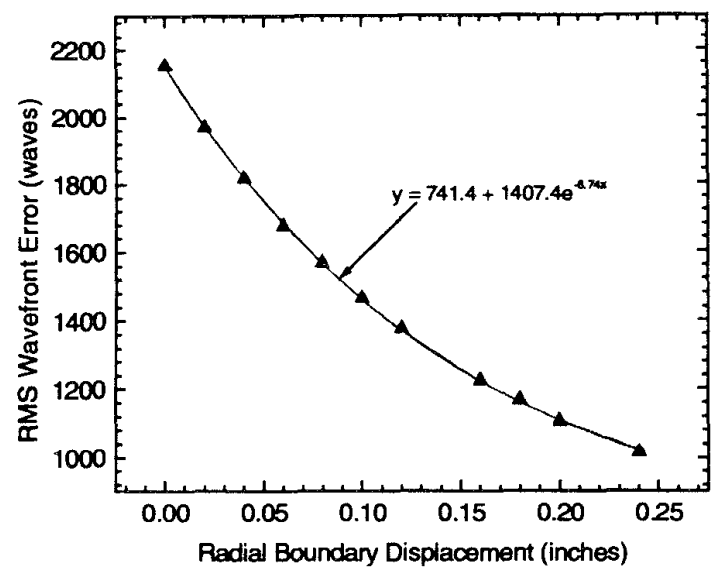

(a)

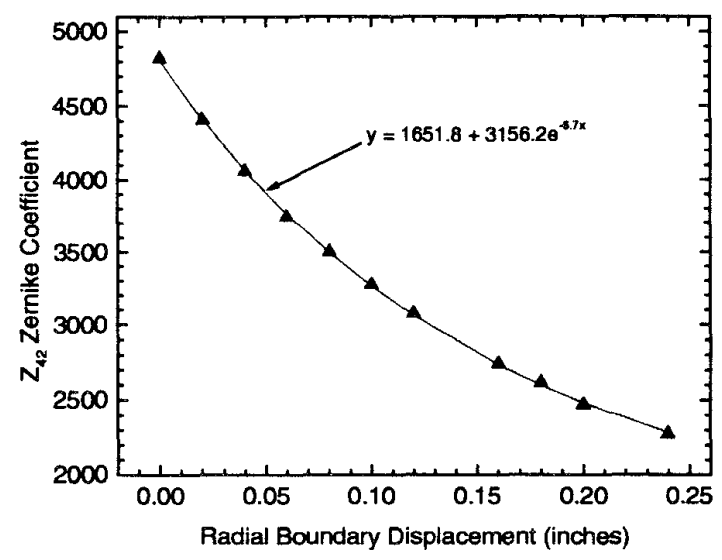

(b)

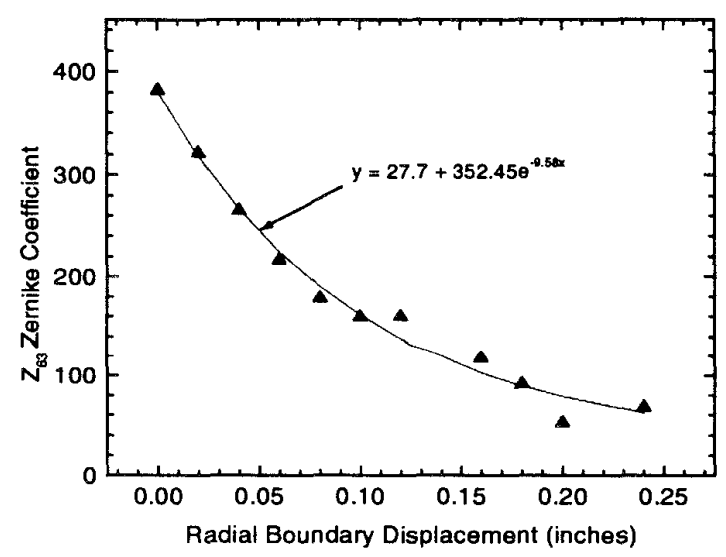

(c)

Figure 6 (a) The rms wavefront error, (b) $Z_{42}$ Zernike coefficient, (c) $\mathrm{Z}_{63}$ Zernike coefficient versus radial boundary displacement for the $f / 1.06$ membrane mirror.
Second, the primary aberration is third order spherical aberration which is a well known aberration. This aberration could be corrected, at least partially, by the other optics in the imaging system. The secondary and tertiary mirrors could be constructed with an aspheric shape which removes the aberration. Unfortunately, this technique would have several limitations. The membrane mirror would be required to have a fixed f-number since the secondary optics would be specifically designed for a certain surface shape of the membrane. Also, the secondary optics would be extremely expensive to manufacture, and these optics would be very sensitive to alignment. Finally, other techniques such as a non-uniform membrane thickness and non-uniform pressure distribution could be incorporated in the membrane mirror to further reduce the aberrations. By manufacturing a membrane which has a variable thickness, the thicker portions of the membrane would be deformed differently thus affecting the surface figure of the membrane.

\section{CONCLUSIONS}

In this paper, it was shown that the Poisson's ratio of a material affects the performance of a membrane mirror, and materials with $v=0.45$ or greater are better suited for use in membrane mirror systems. The imaging capability of membrane mirrors is extremely poor thus requiring techniques to be employed which will improve the optical performance. One technique shown to have the capability to improve the imaging capabilities of the membrane mirror is uniform radial boundary displacement. This technique demonstrated the capability to reduce the rms wavefront error by $65.6 \%$ for an $\mathrm{f} / 1.06$ membrane mirror and $78.7 \%$ for an $f / 1.9$ mirror. This suggests that longer focal length membrane mirrors can receive more benefit from radial boundary displacement. The primary aberration, even offaxis, for these membrane mirrors was found to be $3^{\text {rd }}$ order spherical aberration.

Even though the rms wavefront error is reduced by $65.6 \%$ and greater, the 42 inch membrane mirrors are left with 240 waves or more of aberration depending on the focal length of the mirror. This is an unacceptable amount of aberration for membrane mirrors to be useful. Other techniques such as non-uniform membrane thickness and non-uniform pressure distribution could potentially reduce the aberrations further. Even with these techniques, membrane mirrors will probably never reach diffraction limited performance. However, it is believed that diffraction limited performance is not necessary for membrane mirrors with recent advances in compensated imaging techniques[1,6]. In Gruneisen, et. al.[1], a six inch diameter, four micron thick, nitrocellulose f/6 membrane mirror with $>200$ waves peak to valley wavefront error was corrected to 1.6 times diffraction limited. Even with advances such as these and specially designed secondary optics, the rms wavefront error needs to be improved to less than a few hundred waves for a large diameter (greater than 5 meters) membrane mirror to be useful in large aperture imaging systems. This performance has not yet been demonstrated, and will require the use of radial boundary displacement combined with the other techniques mentioned earlier. 
Future work will include experimental validation of the results presented here. A flexible mirror test cell has been constructed which has multiple pressure chambers and the capability to test the effects of uniform radial boundary displacement. Also, the finite element model will be utilized to study the effects of non-uniform membrane thickness and multiple pressure chambers.

\section{REFERENCES}

[1] Mark T. Gruneisen, Kevin W. Peters, and James M. Wilkes, "Compensated Imaging by Real-Time Holography with Optically Addressed Liquid-Crystal Spatial Light Modulators", SPIE 42 ${ }^{\text {nd }}$ Annual Meeting, San Diego, CA, July 27-Aug 1, 1997, Paper 3143-25 (invited).

[2] H. Hencky, "Uber den Spannungzustand in kreisrunden Platten mit verschwindender Biegungssteiffigkeit", Zeitshrift fur Mathematik und Physik, Vol. 63, pp. 311-317, 1915.

[3] J.D. Campbell, "On the theory of initially tensioned circular membranes subjected to uniform pressure", Quarterly Journal of Mechanics and Applied Mathematics, vol. 9, part 1, pp. 84-93, 1956.

[4] Herbert H. Stevens, Jr., "Behavior of circular membranes stretched above the elastic limit by air pressure", Experimental Stress Analysis, Proceedings of the Society for Experimental Stress Analysis, Vol. II, Number 1, meetings held at the Hotel Statler, Boston, MA, May 18$20,1944$.

[5] Daniel Malacara and Zacarias Malacara, Handbook of Lens Design, New York: Marcel Dekker, Inc., 1994.

[6] Gordon D. Love, "Wave-front correction and production of Zernike modes with a liquid-crystal spatial light modulator", Applied Optics, Vol. 36, No. 7, 1 March 1997.

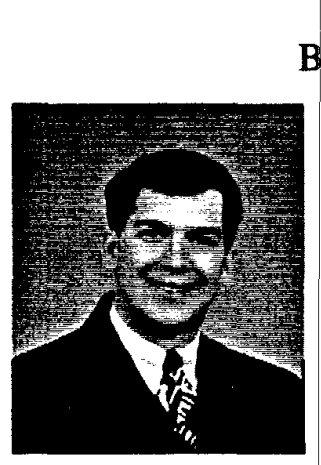

\section{BIOGRAPHIES}

Kevin W. Peters is a research physicist at the Air Force Research Laboratory. He received a B.S. in applied physics from the United States Air Force Academy in 1993 and an M.S. in engineering physics from the Air Force Institute of Technology in 1994. He is a captain in the U.S. Air Force and is currently assigned to the Spectral Sensing Support group at the Air Force Research Laboratory.

Jonathan A. Bishop is a space systems research engineer in the Vehicle Technologies Branch of the Air Force Research Laboratory. He received a B.S. in Aerospace Engineering in 1993 and an M.S. in Mechanical Engineering in 1995, both from the University of Oklahoma. He is a $1^{\text {st }}$ lieutenant in the U.S. Air Force and manages the Space Vehicle Directorate's inflatable structures program.

James M. Wilkes is a research physicist in the Directed Energy Directorate of the Air Force Research Laboratory. He holds an M.S. in applied mathematics and Ph.D. in theoretical physics, both from New Mexico State University. His current research interests include the application of exterior differential forms to contiuum mechanics in general, and to the theory of pressurized membranes in particular, and applications of the vector theory of diffraction to diffraction by devices such as optically addressed spatial light modulators using liquid crystals. He has authored or coauthored over thirty technical papers in the fields of general relativity, astrophysics, electromagnetic theory, and mathematical geodesy. 